\title{
The approximation of entropy for Smoluchowski coagulation equation with TEMOM
}

\author{
Mingliang Xie ${ }^{1 *}$
}

1 State Key Laboratory of Coal Combustion, Huazhong University of Science and Technology, Wuhan 430074, China

* Correspondence: mlxie@mail.hust.edu.cn

\begin{abstract}
In this paper, the definition of information entropy of Smoluchowski coagulation equation for Brownian motion is introduced based on coagulation probability. The expression of entropy is the function of geometric average particle volume and standard deviation with lognormal distribution assumption. The asymptotic solution with moment method shows that the entropy is a monotone increasing function of time, which is equivalence to the entropy based on particle size distribution. the result reveals that the present definition of entropy of Smoluchowski coagulation equation are inadequate because the particle average volume at equilibrium cannot be determined from the principle of maximum entropy. This provides a basis for further exploring the global properties of Smoluchowski coagulation equation.
\end{abstract}

Keywords: information entropy; Brownian coagulation; moment method; coagulation probability; particle size distribution

\section{Introduction}

Brownian motion refers to the continuous random movement or diffusion of particle suspended in a fluid. Brownian aggregation occurs as result of their random motion when particle collide and stick together. Coagulation is regarded as special case of aggregation where there is instantaneous coalescence of particle after collision.

The coagulation kinetics of particles determines the aggregate size distribution. It is fundamental interest in colloid science, with important implications for engineering problems such as the control of water and air pollution. The theoretical treatment of coagulation is described as the Smoluchowski equation as [1]:

$$
\frac{d n}{d t}=\frac{1}{2} \int_{0}^{v} \beta\left(v_{1}, v-v_{1}\right) n\left(v_{1}\right) n\left(v-v_{1}\right) d v_{1}-\int_{0}^{\infty} \beta\left(v_{1}, v\right) n\left(v_{1}\right) n(v) d v_{1}
$$

In which $\mathrm{n}$ is the number of particles per unit spatial volume with particle volume $\mathrm{v}$ at time $t$, and $\beta$ is the collision frequency function which depended on the particle sizes, particle densities, and transport mechanisms in the system. An expression for collision frequency function in the free molecular regime was derived in the gas kinetic approach assuming rigid elastic spheres as

$$
\beta=B_{1}\left(\frac{1}{v_{1}}+\frac{1}{v_{2}}\right)^{1 / 2}\left(v_{1}^{1 / 3}+v_{2}^{1 / 3}\right)^{2}
$$

Where $B_{1}=(3 / 4 \pi)^{1 / 6}\left(6 k_{B} T / \varrho_{p}\right), k_{B}$ is the Boltzmann constant, $T$ is the temperature, and $Q_{p}$ is the particle density.

The expression for the collision frequency in the continuum regime was obtained by solving the diffusion equation for particles around one particle that is assumed to be fixed using the relative diffusion coefficient as 


$$
\beta=B_{2}\left(\frac{1}{v_{1}^{1 / 3}}+\frac{1}{v_{2}^{1 / 3}}\right)\left(v_{1}^{1 / 3}+v_{2}^{1 / 3}\right)
$$

Where $B_{2}=2 k_{B} T / 3 \mu$, and $\mu$ is viscosity of fluid.

Generally, the Smoluchowski coagulation equation with such physically realistic kernel are not solvable due to its nonlinear integro-differential structure. Usually, it can be solved numerically either by deterministic bin methods and moment-based method or stochastic Monte Carlo method. Because of the relative simplicity of implementation and low computational costs, the moment method has become a powerful tool for investigating aerosol microphysical processes for many applications.

The k-th order particle moment is defined as

$$
M_{k}=\int_{0}^{\infty} v^{k} n(v) d v
$$

Especially, $\mathrm{M}_{0}, \mathrm{M}_{1}, \mathrm{M}_{2}$ represent the total particle number concentration, the total particle volume fraction, and the dispersity of particle size distribution, respectively. In a spatially homogeneous system, the particle moments evolve in time can be expressed as

$$
\frac{d M_{k}}{d t}=\frac{1}{2} \int_{0}^{\infty} \int_{0}^{\infty}\left[\left(v_{1}+v_{2}\right)^{k}-v_{1}^{k}-v_{2}^{k}\right] \beta\left(v_{1}, v_{2}\right) n\left(v_{1}, t\right) n\left(v_{2}, t\right) d v_{1} d v_{2}
$$

The minimum set of moments required to close the particle moment equations is the first three. Ten year ago, Yu and Lin [2] proposed Taylor-series expansion method of moment (TEMOM) to approximate Smoluchowski equation. This approach makes no prior assumption on the shape of particle size distribution; the derivation is completely based Taylor series expansion; and the form of TEMOM is relatively simple to be solved asymptotically and analytically $[3,4]$. Therefore, TEMOM not only has advantages in the speed and accuracy of computation, it also provides a new way to analyze the characteristic of Smoluchowski equation theoretically.

\section{The TEMOM model for Brownian coagulation}

In mathematics, a Taylor series is a representation of a function as an infinite sum of terms that are calculated from the values of the function's derivatives at an expansion point (in TEMOM, the expansion point is the mean particle volume $\mathrm{u}=\mathrm{M}_{1} / \mathrm{M}_{0}$ ). In the derivation of TEMOM model, Taylor series approximations are introduced twice, one is the approximation of nonlinear collision frequency function [5], i.e.,

$$
\begin{aligned}
\beta\left(v_{i}, v_{j}\right) & \approx \beta(u, u)+\frac{\partial \beta(u, u)}{\partial v_{i}}\left(v_{i}-u\right)+\frac{\partial \beta(u, u)}{\partial v_{j}}\left(v_{j}-u\right) \\
& +\frac{1}{2} \frac{\partial^{2} \beta(u, u)}{\partial v_{i}^{2}}\left(v_{i}-u\right)^{2}+\frac{\partial^{2} \beta(u, u)}{\partial v_{i} \partial v_{j}}\left(v_{i}-u\right)\left(v_{j}-u\right)+\frac{1}{2} \frac{\partial^{2} \beta(u, u)}{\partial v_{j}{ }^{2}}\left(v_{j}-u\right)^{2}
\end{aligned}
$$

The other is the approximation of higher and fractional order moment as

$$
M_{k}=\frac{M_{1}^{k}}{M_{0}{ }^{k-1}}\left[1+\frac{k(k-1)\left(M_{C}-1\right)}{2}\right]
$$

where the dimensionless particle moment is defined as

$$
M_{C}=\frac{M_{0} M_{2}}{M_{1}^{2}}
$$

In the free molecular regime, the TEMOM model is 


$$
\begin{aligned}
\frac{d M_{0}}{d t} & =\frac{\sqrt{2} B_{1}\left(65 M_{C}^{2}-1210 M_{C}-9223\right) M_{0}^{2}}{5184} \frac{M_{1}^{1 / 6}}{M_{0}^{1 / 6}} \\
\frac{d M_{1}}{d t} & =0 \\
\frac{d M_{2}}{d t} & =-\frac{\sqrt{2} B_{1}\left(701 M_{C}^{2}-4210 M_{C}-6859\right) M_{1}^{2}}{2592} \frac{M_{1}^{1 / 6}}{M_{0}^{1 / 6}}
\end{aligned}
$$

In the continuum regime, the corresponding model is

$$
\begin{aligned}
& \frac{d M_{0}}{d t}=\frac{B_{2}}{81}\left(2 M_{C}{ }^{2}-13 M_{C}-151\right) M_{0}^{2} \\
& \frac{d M_{1}}{d t}=0 \\
& \frac{d M_{2}}{d t}=-\frac{2 B_{2}}{81}\left(2 M_{C}{ }^{2}-13 M_{C}-151\right) M_{1}^{2}
\end{aligned}
$$

It should be point out that $\mathrm{M}_{1}$ remains constant due to the mass conservation.

\section{The information entropy of Smoluchowski coagulation equation}

Following Gmachowski [6], the coagulation probability based on collision frequency is expressed as

$$
p=\frac{\beta n\left(v_{1}\right) n\left(v_{2}\right)}{\int_{0}^{\infty} \int_{0}^{\infty} \beta n\left(v_{1}\right) n\left(v_{2}\right) d v_{1} d v_{2}}
$$

And the normalized particle size distribution is noted as

$$
f(v)=\frac{n(v)}{\int_{0}^{\infty} n(v) d v}=\frac{n(v)}{M_{0}}
$$

Therefore, the corresponding Shannon information entropy is

$$
\begin{aligned}
S & \propto-\int_{0}^{\infty} \int_{0}^{\infty} p \ln p d v_{1} d v_{2} \\
& =-\int_{0}^{\infty} \int_{0}^{\infty} \frac{\beta n\left(v_{1}\right) n\left(v_{2}\right)}{\int_{0}^{\infty} \int_{0}^{\infty} \beta n\left(v_{1}\right) n\left(v_{2}\right) d v_{1} d v_{2}} \ln \frac{\beta n\left(v_{1}\right) n\left(v_{2}\right)}{\int_{0}^{\infty} \int_{0}^{\infty} \beta n\left(v_{1}\right) n\left(v_{2}\right) d v_{1} d v_{2}} d v_{1} d v_{2}
\end{aligned}
$$

For simplicity, the symbols for the integrations are introduced as

$$
I_{k}=\int_{0}^{\infty} v^{k} f(v) \ln f(v) d v
$$

And the following approximation of nonlinear kernel function is performed 


$$
\begin{aligned}
& \beta\left(v_{i}, v_{j}\right) \ln \beta\left(v_{i}, v_{j}\right) \approx \beta(u, u) \ln \beta(u, u) \\
& +\left[\frac{\partial \beta(u, u)}{\partial v_{i}}+\frac{\partial \beta(u, u)}{\partial v_{i}} \ln \beta(u, u)\right]\left(v_{i}-u\right)+\left[\frac{\partial \beta(u, u)}{\partial v_{j}}+\frac{\partial \beta(u, u)}{\partial v_{j}} \ln \beta(u, u)\right]\left(v_{j}-u\right) \\
& +\frac{1}{2}\left[\frac{\partial^{2} \beta(u, u)}{\partial^{2} v_{i}}+\frac{1}{\beta(u, u)}\left(\frac{\partial \beta(u, u)}{\partial v_{i}}\right)^{2}+\frac{\partial^{2} \beta(u, u)}{\partial^{2} v_{i}} \ln \beta(u, u)\right]\left(v_{i}-u\right)^{2} \\
& +\left[\frac{\partial^{2} \beta(u, u)}{\partial v_{i} \partial v_{j}}+\frac{1}{\beta(u, u)}\left(\frac{\partial \beta(u, u)}{\partial v_{i}} \frac{\partial \beta(u, u)}{\partial v_{j}}\right)+\frac{\partial^{2} \beta(u, u)}{\partial v_{i} \partial v_{j}} \ln \beta(u, u)\right]\left(v_{i}-u\right)\left(v_{j}-u\right) \\
& +\frac{1}{2}\left[\frac{\partial^{2} \beta(u, u)}{\partial^{2} v_{j}}+\frac{1}{\beta(u, u)}\left(\frac{\partial \beta(u, u)}{\partial v_{j}}\right)^{2}+\frac{\partial^{2} \beta(u, u)}{\partial^{2} v_{j}} \ln \beta(u, u)\right]\left(v_{j}-u\right)^{2}
\end{aligned}
$$

In the free molecule regime, the denominator in the coagulation probability can be expressed as

$$
\int_{0}^{\infty} \int_{0}^{\infty} \beta n\left(v_{1}\right) n\left(v_{2}\right) d v_{1} d v_{2}=C_{1} \frac{M_{1}^{1 / 6}}{M_{0}^{1 / 6}} M_{0}^{2}
$$

where $\mathrm{C}_{1}$ is noted as

$$
C_{1}=-\frac{\sqrt{2} B_{1}\left(65 M_{C}{ }^{2}-1210 M_{C}-9223\right)}{2592}
$$

Then the entropy can be calculated as

$$
\begin{aligned}
S \propto \ln \left(\frac{C_{1}}{B_{1}} u^{1 / 6}\right) & -2 \sqrt{2} \frac{B_{1}}{C_{1}}\left[2 \ln \left(4 \sqrt{2} u^{1 / 6}\right)+\left(\frac{2}{9}+\frac{5}{24} \ln \left(4 \sqrt{2} u^{1 / 6}\right)\right)\left(M_{C}-1\right)\right] \\
& -2 \sqrt{2} \frac{B_{1}}{C_{1}}\left[4 I_{0}+\frac{1}{3}\left(\frac{1}{u} I_{1}-I_{0}\right)+\frac{5}{24}\left(\frac{1}{u^{2}} I_{2}-\frac{2}{u} I_{1}+M_{C} I_{0}\right)\right]
\end{aligned}
$$

Analogously, the denominator in the coagulation probability in the continuum regime can be calculated as

$$
\int_{0}^{\infty} \int_{0}^{\infty} \beta n\left(v_{1}\right) n\left(v_{2}\right) d v_{1} d v_{2}=C_{2} M_{0}^{2}
$$

where $\mathrm{C}_{2}$ is noted as

$$
C_{2}=-\frac{2 B_{2}}{81}\left(2 M_{C}^{2}-13 M_{C}-151\right)
$$

And the corresponding entropy become

$$
\begin{array}{r}
S \propto \ln \left(\frac{C_{2}}{B_{2}}\right)-2\left(\frac{B_{2}}{C_{2}}\right)\left[4 \ln 2+\frac{1}{9}(1+2 \ln 2)\left(M_{C}-1\right)\right] \\
-2\left(\frac{B_{2}}{C_{2}}\right)\left[4 I_{0}+\frac{1}{9}\left(\frac{1}{u^{2}} I_{2}-\frac{2}{u} I_{1}+M_{C} I_{0}\right)\right]
\end{array}
$$

\section{The asymptotic solution of entropy}

The lognormal function is considered as a good approximation of the particle size distribution, which takes in the following form

$$
f(v)=\frac{n}{M_{0}}=\frac{1}{3 \sqrt{2 \pi} \ln \sigma} \exp \left[-\frac{\ln ^{2}\left(v / v_{g}\right)}{18 \ln ^{2} \sigma}\right] \frac{1}{v}
$$


There are some relationships between the parameters (the geometric average particle volume $\mathrm{vg}$ and standard deviation $\sigma$ ) and the first three particle moments

$$
v_{g}=\frac{M_{1}^{2}}{M_{0}^{3 / 2} M_{2}^{1 / 2}}=\frac{1}{\sqrt{M_{C}}} u
$$

and

$$
\ln ^{2} \sigma=\frac{1}{9} \ln M_{C}
$$

With the lognormal distribution assumption, the integration for $\mathrm{I}_{\mathrm{k}}$ can be calculated as

$$
\begin{aligned}
& I_{0}=-\left[\ln \left(\sqrt{2 \pi \ln M_{C}}\right)+\ln \frac{u}{\sqrt{M_{C}}}+\frac{1}{2}\right] \\
& I_{1}=-u\left(-I_{0}+\frac{3}{2} \ln M_{C}\right) \\
& I_{2}=-u^{2} M_{C}\left(-I_{0}+4 \ln M_{C}\right)
\end{aligned}
$$

It can be found that the integration $I_{k}$ is only the function of geometry average volume and variance (or mean particle volume and dimensionless particle moment)

In the free molecule regime, the asymptotic solution of dimensionless particle moment is constant as $M_{c}=2.2001$, and the mean particle volume evolves with time as

$$
u=3.1918 B_{1}^{6 / 5} M_{1}^{6 / 5} t^{6 / 5}
$$

In the continuum regime, the asymptotic solution of dimensionless particle moment is constant as $\mathrm{Mc}_{\mathrm{c}}=2$, and the mean particle volume evolves with time as

$$
u=\frac{169}{81} B_{2} M_{1} t
$$

Therefore, the entropy is only the function of mean particle volume

$$
S \propto \ln u
$$

And it is a strictly monotone increasing function of time.

Analogously, the growth rate for $\mathrm{I}_{k}$ are approximated as

$$
\begin{aligned}
& \frac{d I_{0}}{d t} \sim-\frac{1}{u} \frac{d u}{d t} ; \\
& \frac{d}{d t}\left(\frac{I_{1}}{u}\right) \sim \frac{d I_{0}}{d t} ; \\
& \frac{d}{d t}\left(\frac{I_{2}}{u^{2}}\right) \sim \frac{d I_{0}}{d t} ;
\end{aligned}
$$

Then the growth rate of entropy can be simplified as

$$
\frac{d S}{d t} \sim \frac{1}{u} \frac{d u}{d t}=\frac{1.2}{t}
$$

In the free molecular regime, and

$$
\frac{d S}{d t} \sim \frac{1}{u} \frac{d u}{d t}=\frac{1}{t}
$$

In the continuum regime, respectively.

For the convenience of analysis, the collision frequency function can be replaced by a constant, i.e., $\beta=C$. In fact, the constant collision kernel is a good approximation of collision frequency function for Brownian coagulation [7], then the coagulation probability become 


$$
p=\frac{\beta n\left(v_{1}\right) n\left(v_{2}\right)}{\int_{0}^{\infty} \int_{0}^{\infty} \beta n\left(v_{1}\right) n\left(v_{2}\right) d v_{1} d v_{2}}=\frac{C n\left(v_{1}\right) n\left(v_{2}\right)}{C M_{0} M_{0}}=f\left(v_{1}\right) f\left(v_{2}\right)
$$

And the entropy becomes

$$
S \propto-\int_{0}^{\infty} \int_{0}^{\infty} p \ln p d v_{1} d v_{2}=-2 \int_{0}^{\infty} f \ln f d v=-2 I_{0}
$$

which shows that the entropy based on coagulation probability and collision frequency is equivalent to that based on particle size distribution. This relationship will greatly simplify the calculation of entropy of Smoluchowski coagulation equation.

The maximum entropy is determined imposing the condition

$$
\frac{\partial S}{\partial M_{C}}=\frac{1}{2} \frac{1}{2 \pi \ln M_{C}} \frac{2 \pi}{M_{C}}-\frac{1}{2} \frac{1}{M_{C}}=0
$$

Which gives

$$
M_{C}=e
$$

And the corresponding standard deviation is $\sigma=1.3956$. The value is very closed to the asymptotic and analytical solution of Smoluchowski coagulation equation with Taylor series expansion method of moment [3,4] and log skew normal distribution [8]. But the mean particle volume cannot be obtained from the principle of maximum entropy. As a distribution at equilibrium, the mean particle volume is an important parameter, which will be determined by the thermodynamics constraint [9]. The result reveals that the present definition of entropy is inadequate.

\section{Results}

In this paper, the information entropy of Smoluchowski coagulation equation for Brownian motion is introduced based on coagulation probability and collision frequency. The asymptotic solutions of entropy together with its growth rate are obtained based on Taylor series expansion method of moment and lognormal distribution assumption. According to the analysis, the definition of entropy based on coagulation probability is equivalent to that based on particle size distribution.

The maximum entropy principle was formulated as a variational principle for determining the probability distribution under constraints. Taking into account that the lognormal distribution was sometimes used as an approximation to the self-preserving size distribution. And self-preserving size distribution may be thought as an equilibrium distribution. The results show that entropy is a strictly monotone increasing function of time. There is no local extremum in the coagulation processes. Therefore, the geometric average volume of equilibrium distribution cannot be determined with maximum entropy principle, and the definition of entropy based on coagulation probability together with that based on particle size distribution are inadequate. In order to determine the mean volume of self-preserving size distribution, it is necessary to explore other definitions of entropy of Smoluchowski equation.

\section{Conclusions}

This section is not mandatory but can be added to the manuscript if the discussion is unusually long or complex.

Funding: This research was funded by the National Natural Science Foundation of China, grant number 11972169 and 11572138.

Institutional Review Board Statement:

Not applicable.

Informed Consent Statement: 
Not applicable.

Data Availability Statement: The study did not report any data.

Acknowledgments: the authors should appreciate the helpful discussion from Prof. Yu Mingzhou at China Jiliang University and Prof. Wang Lian-Ping at University of Delaware and Prof. Alexander Shchekin at St. Petersburg State University.

Conflicts of Interest: The authors declare no conflict of interest.

\section{Appendix A}

The nonlinear collision frequency function in the free molecular regime is approximated as

$$
\begin{aligned}
& \frac{\beta}{B_{1}}=\frac{4 \sqrt{2}}{\sqrt{u}} u^{2 / 3}+\frac{1}{3} \frac{\sqrt{2}}{\sqrt{u} u^{1 / 3}}\left(v_{1}-u\right)+\frac{1}{3} \frac{\sqrt{2}}{\sqrt{u} u^{1 / 3}}\left(v_{2}-u\right) \\
& +\frac{5}{24} \frac{\sqrt{2}}{\sqrt{u} u^{4 / 3}}\left(v_{1}-u\right)^{2}-\frac{25}{36} \frac{\sqrt{2}}{\sqrt{u} u^{4 / 3}}\left(v_{1}-u\right)\left(v_{2}-u\right)+\frac{5}{24} \frac{\sqrt{2}}{\sqrt{u} u^{4 / 3}}\left(v_{2}-u\right)^{2}
\end{aligned}
$$

And the nonlinear integration kernel is approximated as

$$
\begin{aligned}
& \left(\frac{\beta}{B_{1}}\right) \ln \left(\frac{\beta}{B_{1}}\right)=\frac{4 \sqrt{2}}{\sqrt{u}} u^{2 / 3} \ln \left(\frac{4 \sqrt{2}}{\sqrt{u}} u^{2 / 3}\right) \\
& +\left(\frac{1}{3} \frac{\sqrt{2}}{\sqrt{u} u^{1 / 3}}+\frac{1}{3} \frac{\sqrt{2}}{\sqrt{u} u^{1 / 3}} \ln \left(\frac{4 \sqrt{2}}{\sqrt{u}} u^{2 / 3}\right)\right)\left(v_{1}-u\right) \\
& +\left(\frac{1}{3} \frac{\sqrt{2}}{\sqrt{u} u^{1 / 3}}+\frac{1}{3} \frac{\sqrt{2}}{\sqrt{u} u^{1 / 3}} \ln \left(\frac{4 \sqrt{2}}{\sqrt{u}} u^{2 / 3}\right)\right)\left(v_{2}-u\right) \\
& +\left(\frac{2}{9} \frac{\sqrt{2}}{\sqrt{u} u^{4 / 3}}+\frac{5}{24} \frac{\sqrt{2}}{\sqrt{u} u^{4 / 3}} \ln \left(\frac{4 \sqrt{2}}{\sqrt{u}} u^{2 / 3}\right)\right)\left(v_{1}-u\right)^{2} \\
& -\left(\frac{2}{3} \frac{\sqrt{2}}{\sqrt{u} u^{4 / 3}}+\frac{25}{36} \frac{\sqrt{2}}{\sqrt{u} u^{4 / 3}} \ln \left(\frac{4 \sqrt{2}}{\sqrt{u}} u^{2 / 3}\right)\right)\left(v_{1}-u\right)\left(v_{2}-u\right) \\
& +\left(\frac{2}{9} \frac{\sqrt{2}}{\sqrt{u} u^{4 / 3}}+\frac{5}{24} \frac{\sqrt{2}}{\sqrt{u} u^{4 / 3}} \ln \left(\frac{4 \sqrt{2}}{\sqrt{u}} u^{2 / 3}\right)\right)\left(v_{2}-u\right)^{2}
\end{aligned}
$$

Similarly, the nonlinear collision frequency function in the continuum regime can be approximated as

$$
\frac{\beta}{B_{2}}=4+\frac{1}{9 u^{2}}\left(v_{1}-u\right)^{2}-\frac{2}{9 u^{2}}\left(v_{1}-u\right)\left(v_{2}-u\right)+\frac{1}{9 u^{2}}\left(v_{2}-u\right)^{2}
$$

And the corresponding nonlinear integration kernel is approximated as

$$
\begin{aligned}
& \frac{\beta}{B_{2}} \ln \frac{\beta}{B_{2}}=8 \ln 2+\frac{1}{9 u^{2}}(1+2 \ln 2)\left(v_{1}-u\right)^{2} \\
& -\frac{2}{9 u^{2}}(1+2 \ln 2)\left(v_{1}-u\right)\left(v_{2}-u\right)+\frac{1}{9 u^{2}}(1+2 \ln 2)\left(v_{2}-u\right)^{2}
\end{aligned}
$$

The integration both in free molecule and continuum regime can be written as

$$
\int_{0}^{\infty} \int_{0}^{\infty} \beta n\left(v_{1}\right) n\left(v_{2}\right) d v_{1} d v_{2}=f\left(M_{0}, M_{0}, M_{0}\right) M_{0}^{2}
$$

Then entropy can be expressed as 


$$
\begin{aligned}
& S \propto-\int_{0}^{\infty} \int_{0}^{\infty} \frac{\beta n\left(v_{1}\right) n\left(v_{2}\right)}{\int_{0}^{\infty} \int_{0}^{\infty} \beta n\left(v_{1}\right) n\left(v_{2}\right) d v_{1} d v_{2}} \ln \frac{\beta n\left(v_{1}\right) n\left(v_{2}\right)}{\int_{0}^{\infty} \int_{0}^{\infty} \beta n\left(v_{1}\right) n\left(v_{2}\right) d v_{1} d v_{2}} d v_{1} d v_{2} \\
& =\ln f\left(M_{0}, M_{0}, M_{0}\right) \\
& -\frac{1}{f\left(M_{0}, M_{0}, M_{0}\right)} \int_{0}^{\infty} \int_{0}^{\infty} \beta \ln \beta g\left(v_{1}\right) g\left(v_{2}\right) d v_{1} d v_{2} \\
& -\frac{1}{f\left(M_{0}, M_{0}, M_{0}\right)} \int_{0}^{\infty} \int_{0}^{\infty} \beta\left[g\left(v_{1}\right) \ln g\left(v_{1}\right)\right] g\left(v_{2}\right) d v_{1} d v_{2} \\
& -\frac{1}{f\left(M_{0}, M_{0}, M_{0}\right)} \int_{0}^{\infty} \int_{0}^{\infty} \beta g\left(v_{1}\right)\left[g\left(v_{2}\right) \ln g\left(v_{2}\right)\right] d v_{1} d v_{2}
\end{aligned}
$$

Substituting the approximation of nonlinear collision frequency function $(\beta)$ and integration kernel $(\beta \ln \beta)$ in to the formula, then the equation (18) and $(21)$ can be obtained.

\section{References}

1. Friedlander S.K. Smoke, dust and haze: fundamentals of aerosol dynamics (2 ${ }^{\text {nd }}$ wd.). London: Oxford University Press, 2000.

2. Yu M.Z.; Lin J.Z.; Chan T.L. A new moment method for solving the coagulation equation for particle in Brownian motion. Aerosol Science and Technology, 2008, 42, 705-713.

3. Xie M.L.; Wang L.P. Asymptotic solution of population balance equation based on TEMOM model. Chemical Engineering Science, 2013, 94, 79-83.

4. Xie M.L., He Q. Analytical solution of TEMOM model for particle population balance equation due to Brownian coagulation. Journal of Aerosol Science, 2013, 66, 24-30

5. Xie M.L. Error estimation of TEMOM for Brownian coagulation. Aerosol Science and Technology, 2016, 50, 919-925

6. Gmachowski L. A method of maximum entropy modeling the aggregation kinetics. Colloids and Surfaces A: Physicochemical and Engineering Aspects, 2001, 176, 151-159.

7. He Q.; Shchekin A.K.; Xie M.L. New analytical TEMOM solution for a class of collision kernels in the theory of Brownian coagulation. Physica A, Statistical Mechanics and Its Applications, 2015, 428, 435-442.

8. Wang K.Y.; Yu S.Y; Peng W. A novel moment method using the log skew normal distribution for particle coagulation. Journal of Aerosol Science, 2019, 134, 95-108.

9. Xie M.L.; Yu M.Z. Thermodynamic analysis of Brownian coagulation based on moment method. International Journal of Heat and Mass Transfer, 2018, 122, 922-928. 